\title{
Type 2 myocardial infarction: a diagnostic and therapeutic challenge in contemporary cardiology
}

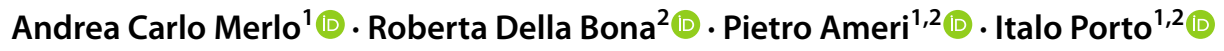

Received: 8 June 2021 / Accepted: 18 December 2021 / Published online: 14 February 2022

(c) The Author(s), under exclusive licence to Società Italiana di Medicina Interna (SIMI) 2022

\begin{abstract}
In the expanding world of cardiovascular diseases, rapidly reaching pandemic proportions, the main focus is still on coronary atherosclerosis and its clinical consequences. However, at least in the Western world, middle-aged male patients with acute myocardial infarction are no more the rule. Due to a higher life expectancy and major medical advances, physicians are to treat older and frailer individuals, usually with multiple comorbidities. In this context, myocardial ischaemia and infarction frequently result from an imbalance between myocardial oxygen supply and demand-i.e., type 2 myocardial infarction (T2MI), according to the current universal definition — rather than coronary atherothrombosis. Moreover, the increasing use of high-sensitivity cardiac troponin assays has led to a heightened detection of T2MI—often causing relatively little myocardial injury—, which seems to have doubled its numbers in recent years. Nevertheless, owing to its multifaceted pathophysiology and clinical presentation, T2MI is still underdiagnosed. Perhaps more importantly, T2MI is also victim of undertreatment, as drugs that constitute the cornerstone of therapy in most cardiovascular diseases are much more unlikely to be prescribed in T2MI than in coronary atherothrombosis. In this paper, we review the recent literature on the classification, pathophysiology, epidemiology, and management of T2MI, trying to summarise the state-of-the-art knowledge about this increasingly important pathologic condition. Finally, based on the current scientific evidence, we also propose an algorithm that may be easily utilised in clinical practice, in order to improve T2MI diagnosis and risk stratification.
\end{abstract}

Keywords Myocardial infarction $\cdot$ Myocardial injury $\cdot$ Cardiac troponin $\cdot$ Coronary angiography

\section{Scope of the problem}

Cardiovascular diseases (CVDs) are the main cause of morbidity and mortality in both sexes in Western countries, responsible for one-third of all deaths worldwide and nearly $50 \%$ of all deaths in Europe-over 4 million each year [1]. Furthermore, in the near future, both the Western world and the developing countries are bracing themselves for a "storm" of CVDs, due to the dramatic prevalence and incidence of obesity and type-2 diabetes mellitus [2]. Amongst

Italo Porto

italo.porto@unige.it

1 Cardiovascular Unit, Department of Internal Medicine and Medical Specialties (DIMI), Chair of Cardiovascular Diseases, University of Genoa, Viale Benedetto XV, 10, 16132 Genoa, Italy

2 Cardiology Unit, DICATOV - Cardiothoracic and Vascular Department, IRCCS Ospedale Policlinico San Martino, Genoa, Italy
CVDs, ischaemic heart disease (IHD) provokes the majority of cardiovascular deaths globally-more than twice that caused by cancer [1]. Specifically, most IHD-related deceases are secondary to sudden cardiac death and acute coronary syndrome (ACS), with about 660.000 individuals having a new heart attack every year in the USA $[1,3]$.

ACS mainly means myocardial infarction (MI) since high-sensitivity cardiac troponin (cTn) assays were introduced, resulting in a $\approx 4 \%$ absolute and $\approx 20 \%$ relative reduction in the diagnosis of unstable angina and a corresponding increase in the detection of MI attributable to acute atherothrombosis-i.e. type $1 \mathrm{MI}$ (T1MI) [4-7]. Also type 2 MI (T2MI), broadly defined as an MI secondary to a myocardial oxygen supply/demand mismatch in the absence of acute atherothrombosis, has become a very common clinical entity, being now detected twice as much as with the old assays $[7,8]$.

Interestingly, however, the heightened diagnostic potential of high-sensitivity cTn has not translated into better outcomes, perhaps because physicians are still reluctant to 
interpret mild cTn elevations as proper MIs and treat them appropriately. This observation is particularly valid for T2MI, as under its umbrella completely different pathological processes fall $[9,10]$. In addition, fewer than two-thirds of patients with T2MI are referred to a cardiology consultation, and most of them do not have any outpatient follow-up after discharge [11].

Due to the complex nature of T2MI, current guidelines for ACS management hardly mention the issue $[12,13]$. Yet, according to available data, T2MI represents at least $26 \%$ of all MIs, even when specific predetermined oxygen mismatch criteria are applied [14]. Besides, the recent spread of coronavirus disease 2019 has enormously increased the incidence of myocardial injury and T2MI, due to the viral effects on the lungs and vessels [15]. Lastly, the incidence of T2MI, already equalling that of T1MI in the community, is poised to grow exponentially in the near future in view of population ageing [16].

According to several observational studies, outcomes appear poorer and in-hospital complications more frequent in T2MI than in T1MI, with short- and intermediate-term mortality rates being near threefold higher and reaching $13.6 \%$ and $23.9 \%$ at 30 days and 1 year, respectively [17, 18]. Such an excess in all-cause death after T2MI mainly results from non-cardiovascular causes [17, 19]. However, cardiovascular mortality explains up to $43 \%$ of deaths after T2MI, and major adverse cardiovascular events (MACE), especially if IHD coexists, are as high as after T1MI, thus creating a great opportunity to improve prognosis in highrisk individuals $[17,20]$.

\section{Definition and pathophysiology of T2MI}

According to the Fourth Universal Definition of Myocardial Infarction (UDMI), T2MI is the consequence of an imbalance between myocardial oxygen supply and demand unrelated to acute coronary atherothrombosis [7]. Therefore, T2MI can have extremely variable aetiologies-from spontaneous coronary artery dissection (SCAD) to anaemia, from a hypertensive crisis to coronary embolism-, making the diagnosis very challenging. T2MI may constitute the main reason leading to clinical presentation-e.g. in case of vasospasm-, but more often is an epiphenomenon of another illness-e.g. pneumonia or a sustained tachyarrhythmia - whose pathophysiological effects determine either a reduced myocardial perfusion/oxygenation or an increased myocardial oxygen demand. If the gap becomes critical, especially when both mechanisms act synergistically, myocardial ischaemia and cardiomyocyte necrosis occur. In addition, the development of MI greatly depends on the magnitude of the stressor, the presence of non-cardiac comorbidities, and the extent of underlying IHD and cardiac structural abnormalities, i.e. the ischaemic threshold [7].

Owing to the considerably variable pathogenesis of T2MI, a partial modification of the current UDMI has recently been proposed [21]. In their article, de Lemos et al. underline that some T2MI aetiologies determine a sudden, clinically evident myocardial oxygen supply/demand mismatch through acute coronary occlusion, thus resembling coronary thrombosis rather than other non-coronary T2MI causes that lead to multi-organ involvement and systemic manifestations. Consequently, their proposal for a revised classification would shift SCAD, coronary embolism, and coronary spasm into T1MI category and rename them T1MI $\mathrm{B}, \mathrm{C}$, and D, respectively. T2MI patients would further be subdivided based on IHD, which significantly affects following management and therapy. Such a proposal pays greater attention to pathophysiology and initial treatment, highlighting that all MIs due to acute coronary occlusion, either thrombotic or not-except for aortic dissection involving the coronary ostia—share important similarities and require prompt myocardial revascularisation whenever possible.

\section{Epidemiology of T2MI}

Distinct pathogeneses imply relevant epidemiological differences between T1MI and T2MI. Firstly, T2MI patients tend to be older, which probably results from a greater susceptibility of elderly people to conditions that may cause an imbalance between myocardial oxygen supply and demand $[19,22,23]$. This relation seems stronger than the mere link between age and IHD. Secondly, in T2MI patients the prevalence of overweight, dyslipidaemia, and smoking - wellknown risk factors for T1MI-is substantially lower [19, $22,23]$. In line with this, prior MI, percutaneous coronary intervention (PCI), and coronary artery bypass graft are more frequent in T1MI [22]. Moreover, most data highlight a larger percentage of women amongst patients with T2MI [19, 22-24]. Alongside the greater susceptibility of men to T1MI, it should be remembered that women are more subject to coronary artery spasm, coronary microvascular dysfunction, and SCAD.

T2MI patients are more likely to display comorbidities such as hypertension, anaemia, valvular heart disease (VHD), atrial fibrillation (AF), heart failure (HF), chronic kidney disease (CKD), liver disease, chronic obstructive pulmonary disease (COPD), peripheral artery disease, depression, alcohol and substance use disorder, a worse functional status, and even a higher Global Registry of Acute Coronary Events (GRACE) risk score at presentation [19, 22, 23]. Indeed, T2MI has traditionally been linked to higher mortality but lower or similar rate of MACE, suggesting that deceases are mainly driven by patients' age and associated 
conditions [25]. Accordingly, a recent observational study by McCarthy et al. on the largest cohort of T2MI patients ever published shows a lower in-hospital mortality in T2MI than in T1MI, after multivariable adjustment [22]. Overall, T2MI population resembles patients with non-ischaemic myocardial injury rather than those with T1MI [9]. T2MI patients also have a distinct humoral footprint, with biomarkers of volume overload, atrial arrhythmias, and systemic infection being higher and more useful for diagnosis and prognostication than in T1MI [26].

The presence of ST-segment elevation (STE) in T2MI, as well as other ischaemic electrocardiogram (ECG) changes and regional wall motion abnormalities (RWMA), is significantly lower than in T1MI [27]. In general, the more severe the ECG changes, the worse the outcomes [28, 29]. An anterior localisation of T1MI is common, whereas the localisation of T2MI is often undetermined [19, 30]. Likely owing to the seasonal trend of respiratory infections, the incidence of T2MI seems to increase from autumn to winter [22]. The occurrence of atypical angina, dyspnoea, and arrhythmias is higher in T2MI patients [19, 30]. Due to its frequently atypical presentation, the prevalence of T2MI is notably greater in all-comers than in chest pain populations [20,31]. Consequently, distinguishing T2MI from both T1MI and myocardial injury is challenging, with low agreement rates amongst trained adjudicators [30, 32].

The two categories of MI also share important features. Above all, IHD plays a pivotal role in both T1MI and T2MI-substrate of atherothrombosis in the former, precipitating factor in the latter. In general, IHD is a common finding in T2MI patients selected for coronary angiography (CA), and carries a worse prognosis [17, 27, 33, 34]. Clearly, in the setting of T2MI, though indirectly involved in its pathogenesis, IHD can worsen myocardial perfusion and induce hypoxia in course of increased oxygen demand. Consistently, in the aforementioned study by McCarthy et al., cardiovascular aetiologies for readmission were common in both T1MI and T2MI patients [22]. The significant rates of MACE after T2MI underline the relevance of cardiac conditions, e.g. fixed IHD, in this population [17, 20].

\section{Diagnosing $\mathrm{T} 2 \mathrm{MI}$ and its subtypes: therapeutic implications}

Sufficient overlap exists that neither cardiovascular risk factors and IHD nor clinical presentation nor cTn values permit a reliable differentiation between T1MI and T2MI [25, 29]. In addition, whilst timely CA may be deemed useless-when the diagnosis of T2MI appears obvious-, it may also be contraindicated-if the patient's conditions are too severe-or inconclusive [7]. Indeed, except when a thrombus causing impaired flow is detected, diagnostic certainty is rare. Sometimes not even the aid of state-of-theart tools for intracoronary imaging-mainly optical coherence tomography - is enough, since ruptured plaques are commonly seen in asymptomatic patients and in those with chronic IHD too. In fact, plaque rupture or erosion may constitute the pathological correlate of T1MI or an occasional finding followed by silent plaque healing and growth [35]. Thus, T2MI is usually a working diagnosis before CA, and might become likely rather than certain after it.

Nonetheless, a correct and definitive identification of this clinical entity is paramount, as the therapeutic management of T2MI significantly differs from that of T1MI in most cases. When T1MI occurs, the principal aim of the physician is to counteract acute thrombosis with drugs inactivating clot formation (aspirin, $\mathrm{P}_{2} \mathrm{Y}_{12}$ inhibitors, anticoagulants, etc.), reducing myocardial ischaemia (beta-blockers, nitrates, etc.), and stabilising atherosclerotic plaques (statins, etc.). Instead, no unique treatment for T2MI exists, since this category includes completely diverse clinical pictures and pathologic processes: it would be pointless treating haemorrhagic anaemia, SCAD, and AF in the same way. The only possible management of T2MI is a causal therapy of its associated conditions through a phenotype-specific approach, once the patient has been stabilised and the trigger has been corrected or controlled [25, 29]. Currently, it is not possible to formulate definite recommendations because of the minimal evidence resulting from observational and retrospective studies, which should be interpreted as hypothesis-generating only $[10,36]$. According to a single-centre study, the use of evidence-based medicines in T2MI can halve mortality, but these data need stronger validation [10].

Specifically, defining the presence of IHD and its burden seems crucial to inform management, albeit complete revascularisation provided no clinical benefit to T2MI patients in the COMPLETE trial [37, 38]. McCarthy et al. found a significantly lower adjusted risk of in-hospital mortality in patients undergoing revascularisation, but all-cause death and readmission for heart failure or MI at 30 days did not differ [22]. The prognostic impact and the cost-effectiveness of systematic risk stratification through CA or coronary computed tomography angiography (CCTA) in T2MI are being studied by the DEMAND-MI (ClinicalTrials.gov NCT03338504) and the ACT-2 trials [39].

Since existing guidelines do not particularly deal with T2MI, the employment of the quality indicators proposed by the European Society of Cardiology (ESC) in its 2020 non-STE ACS guidelines as a means to improve cardiovascular care and outcomes is challenging, as they mainly refer to T1MI. Whilst some are applicable to both diseases-e.g. in-hospital use of high-sensitivity cTn-, others relate to T1MI only-e.g. use of CA within $24 \mathrm{~h}$ and proportion of patients with $\mathrm{P} 2 \mathrm{Y}_{12}$ receptor inhibition [12]. With so many uncertainties about the management of T2MI, validated, 
feasible, concretely interpretable, and specific quality indicators are dramatically needed, in order to improve diagnosis and treatment.

Overall, available data being very scarce, a detailed characterisation of T2MI population is compelling. Clinicians should exclude T2MI mainly by investigating all its potential triggers. Besides, they should not forget non-ischaemic myocardial injury $[25,29,40]$.

\section{A proposal for a diagnostic algorithm}

We have designed a hypothetic algorithm to systematically analyse the clinical picture through a step-by-step approach, once other cardiovascular emergencies-e.g. aortic dissection and pulmonary embolism-have been ruled out (Fig. 1).

Firstly, as soon as the patient arrives at the emergency department, vital signs are monitored and an ECG is recorded. A hypertensive crisis or a paroxysmal arrhythmia, although a possible T1MI presentation as well, immediately suggests the diagnosis. A significant reduction in capillary oxygen saturation-e.g. during COPD exacerbation-may also explain T2MI.

Secondly, urgent blood tests must be performed. Haemoglobin, white blood cells, creatinine, C-reactive protein, and an arterial blood gas test can help physicians assess the presence of anaemia, hypoxaemia, and infections. Globally, the main causes of T2MI include renal failure, infections, sepsis, hypertension, arrhythmias, respiratory

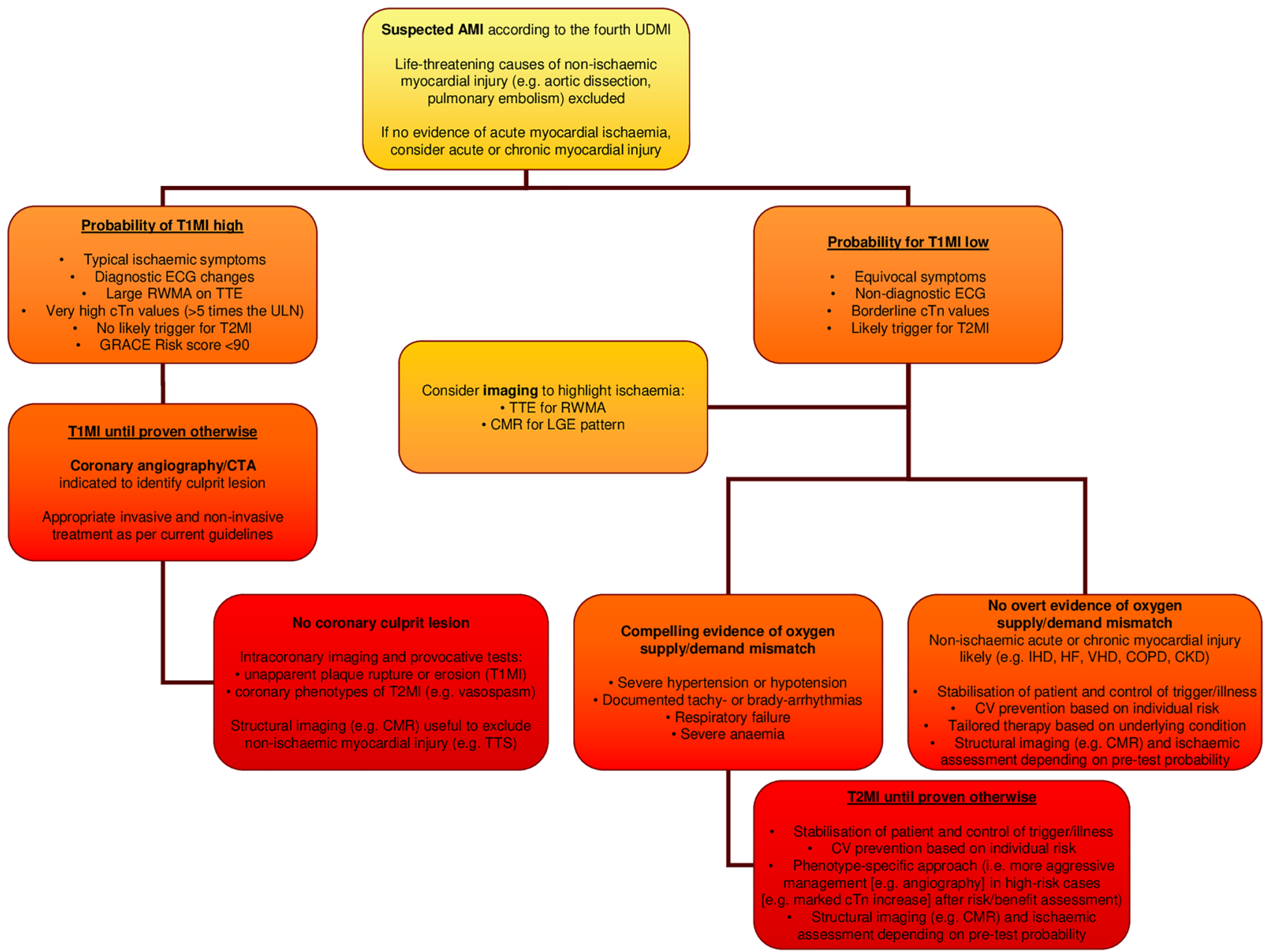

Fig. 1 Algorithm for the practical management of cTn elevations according to patient's risk, based on the current UDMI and the available scientific evidence. $\mathrm{AMI}=$ acute myocardial infarction; $\mathrm{CKD}=$ chronic kidney disease $\mathrm{CMR}=$ cardiac magnetic resonance; $\mathrm{COPD}=$ chronic obstructive pulmonary disease; $\mathrm{CTA}=$ computed tomography angiography; $\mathrm{cTn}=$ cardiac troponin; $\mathrm{CV}=$ cardiovascular; $\mathrm{ECG}=$ electrocardiogram; GRACE $=$ Global Registry of Acute
Coronary Events; $\mathrm{HF}=$ heart failure; $\mathrm{IHD}=$ ischaemic heart disease; LGE = late gadolinium enhancement; RWMA = regional wall motion abnormalities; $\mathrm{T} 1 \mathrm{MI}=$ type 1 myocardial infarction; $\mathrm{T} 2 \mathrm{MI}=$ type 2 myocardial infarction; TTE $=$ transthoracic echocardiography; TTS $=$ takotsubo syndrome UDMI $=$ Universal Definition of Myocardial Infarction; ULN = upper level of normal; VHD= valvular heart disease 
failure, anaemia, and bleeding, with at least one of them affecting the majority of patients $[18,22,41]$.

Then, a relatively simple and quick evaluation enables physicians to exclude most T2MI cases and initiate T1MI therapy, if clinically indicated. Otherwise, it can guide the following diagnostic pathway-e.g. severe iron deficiency anaemia will likely require digestive endoscopy to investigate possible gastrointestinal bleeding.

To provide the algorithm with greater accuracy, we support the use of the GRACE risk score, as significant differences in this parameter between T1MI and T2MI exist $[19,42]$. In one study, the mean values are $110 \pm 35$ and $150 \pm 32$ in T1MI and T2MI, respectively, implying that T2MI patients have a negligible probability of presenting a GRACE risk score $<90$ [19]. This appears reasonable in view of the more severe conditions of T2MI population, reflecting higher heart rates, lower blood pressures, and increased Killip classes. However, although the best available score in the absence of bespoke tools-even superior to the TARRACO score, specifically formulated for T2MI-, the GRACE 2.0 score performed less well in T2MI than in T1MI in one study, likely due to the abovementioned discrepancies [42]. Despite the GRACE risk score being the gold standard for risk stratification, similar considerations may apply to the older and less accurate Thrombolysis in Myocardial Infarction risk score, which is also specific to ACS patients and will likely show higher values in T2MI than in T1MI [43].

We also foster careful consideration of cTn as a quantitative parameter of myocardial necrosis. Indeed, although the overlap in cTn values between T1MI and T2MI hampers differential diagnosis, the magnitude of its elevation may somewhat help the physician [31]. In a small Japanese study, a rise of cTn T $>5$ times the upper level of normal (ULN) before CA had a positive predictive value $>90 \%$ for the presence of coronary thrombus [44]. Excluding those with STE, requiring emergent CA, the ESC guidelines state that patients can be safely discharged after more than $3 \mathrm{~h}$ from symptom onset, if pain-free and with normal baseline cTn values, whereas those with mild cTn elevation should undergo serial measurements, as management depends on the magnitude of cTn increase. Particularly, patients with markedly elevated cTn values-i.e. $>5$ times the ULN_-ought to be admitted to hospital and treated as T1MI until proven otherwise, due to their high pre-test probability [12]. Although cases of extensive T2MI with a substantial muscle loss exist, they are relatively rare and generally affect critically ill patients. Often, T2MI is a secondary manifestation of another illness and determines a mild-to-moderate rise of cTn. Consequently, the detection of significant cardiomyocyte necrosis after few hours from symptom onset potently predicts coronary thrombosis $[25$, 29, 40].
In general, we endorse the application of either the $0 \mathrm{~h} / 1 \mathrm{~h}$ or the $0 \mathrm{~h} / 2 \mathrm{~h}$ algorithm, as recommended by the ESC guidelines, as they constitute quick and easy tools for risk stratification, in order to estimate the likelihood of ACS in such patients and improve diagnosis [12]. Although the algorithm is suggested in T1MI, it may help inform management also in the setting of T2MI, given its correlation with the extent of cardiomyocyte necrosis. Important limits of the said algorithms are their unclear accuracy in T2MI-due to smaller cTn increases than in T1MI-and availability of high-sensitivity cTn assays, which cannot be taken for granted at present [45]. Besides, CKD notably reduces the specificity of the diagnostic workup, as mild increases in cTn may depend on slow plasma clearance instead of ACS, particularly in case of chronic myocardial injury due to coexisting structural cardiac disease. In this context, the assessment of other biomarkers of myocardial necrosis that are less influenced by glomerular filtration rate-above all, creatine-kinase and its myocardial band-becomes pivotal. Overall, the suggested diagnostic tools should always be interpreted with both a detailed clinical assessment and the 12-lead ECG [12, 46].

Undoubtedly, the trickiest clinical scenario is characterised by mild cTn elevation together with atypical symptoms and/or non-diagnostic ECG abnormalities. In such cases, physicians should concentrate their efforts on gathering objective documentation of acute myocardial ischaemia, principally through imaging [40]. Accordingly, either T2MI or non-ischaemic myocardial injury may be diagnosed [25, $29,40]$.

Thus, when the diagnosis remains unclear at the end of the suggested algorithm (Fig. 1), clinical history and physical examination should guide further diagnostic workup together with echocardiography and cardiac magnetic resonance (CMR), which are useful tools to highlight signs of ongoing or past myocardial ischaemia-e.g. RWMA. However, diagnostic performance of common non-invasive tests is better in case of myocardial ischaemia with a regional distribution-e.g. due to coronary spasm-than when mild and diffuse myocardial ischaemia occurs-e.g. following a hypertensive crisis [47, 48]. Therefore, despite the value of echocardiography and CMR for the detection of myocardial ischaemia, we are aware that they may also turn out inconclusive.

A history of HF, AF, COPD, or CKD, as well as imaging evidence of left ventricular hypertrophy or severe VHD, may contribute to the diagnosis of T2MI or non-ischaemic myocardial injury. In the latter case, CMR proves essential for tissue characterisation and estimation of cardiac function. Moreover, biomarkers of myocardial wall stretch-e.g. brain natriuretic peptide and its $\mathrm{N}$-terminal precursor-, atrial arrhythmias-e.g. midregional proadrenomedullin-, and neuro-hormonal and inflammatory activation-e.g. copeptin and procalcitonin-may suggest the aetiology of T2MI/ 
non-ischaemic myocardial injury or reveal important comorbidities/complications [26].

As reported above, known IHD-particularly if multivessel-heightens the risk of T2MI. Therefore, the patient stabilised and the trigger controlled, either invasive or noninvasive ischaemic assessment after T2MI is reasonable, based on pre-test probability. Particularly, the ISCHAEMIA trial has recently shown the relevance of knowing the burden and localisation of coronary atherosclerosis [49]. In this regard, CCTA is suitable for low- and intermediate-risk patients, as its main strength is a high negative predictive value. Conversely, high-risk patients are unlikely to benefit from a non-invasive management, as their great likelihood of IHD as well as their possible comorbidities-e.g. CKD, AF, HF-limits the accuracy of CCTA and renders CA mandatory most of the time. However, selected patients with a moderate pre-test probability are reasonable candidates for $\mathrm{CA}$, in order to immediately undergo PCI if necessary and receive a lower dose of contrast medium. Besides, correct interpretation of CCTA requires significant expertise, which may not be available locally. Overall, considering its limited cost, wide availability, and very low complication rates, in our opinion CA constitutes the gold standard for risk stratification of T2MI patients with suspected concomitant IHD.

Performing CA requires assessment of bleeding risk, which may sometimes overcome ischaemic risk in T2MI subpopulations. With respect to ACS patients, the CRUSADE score was formulated to stratify the risk of major bleeding before CA [50]. However, it dates back to more than a decade ago, when a trans-femoral approach was preferred over a trans-radial one, catheters employed had bigger sizes, upstream administration of glycoprotein IIb/ IIIa inhibitors was frequent, and oral anticoagulation-if appropriate-centred on vitamin K antagonists. In 2019, the Academic Research Consortium for High Bleeding Risk identified a list of criteria to define patients at risk of major bleeding among those referred to PCI [51]. Although possibly useful for the evaluation of haemorrhagic risk, these tools do not completely reflect state-of-the-art clinical practice and currently have a limited prognostic power in T1MI. Notwithstanding, the assessment of bleeding risk plays a fundamental role in T2MI, owing both to the absence of coronary atherothrombosis and to the greater complexity of these patients. Therefore, the use of such scores in T2MI, though not yet validated, may provide incremental value and inform treatment-e.g. optimal timing for CA.

Notably, T2MI and MINOCA are deeply related, as particularly young patients and those with fewer comorbidities may not have IHD. As stated by the 2020 ESC non-STE ACS guidelines, all MINOCA patients should undergo CMR in the absence of an obvious underlying cause [12]. Accordingly, we strongly support the use of CMR in these patients, as we think that cardiomyocyte necrosis of ischaemic origin, even in the absence of significant IHD, requires further investigation.

Future studies are warranted with the aim of improving our knowledge of T2MI, defining objective diagnostic criteria, and permitting an indisputable diagnosis. Randomised prospective trials of T2MI secondary prevention are also needed, in order to clarify whether improved diagnosis can finally improve outcomes [9].

Funding The authors did not receive support from any organization for the submitted work.

\section{Declarations}

Conflict of interest The authors declare that they have no conflict of interest.

Ethical approval The manuscript does not contain clinical studies or patient data.

Informed Consent For this type of study, formal consent is not required.

\section{References}

1. Townsend N, Wilson L, Bhatnagar P, Wickramasinghe K, Rayner M, Nichols M (2016) Cardiovascular disease in Europe: epidemiological update 2016. Eur Heart J 37(42):3232-3245

2. O'Keeffe C, Kabir Z, O'Flaherty M, Walton J, Capewell S, Perry IJ (2013) Modelling the impact of specific food policy options on coronary heart disease and stroke deaths in Ireland. BMJ Open 3(7)

3. Mozaffarian D, Benjamin EJ, Go AS, Arnett DK, Blaha MJ, Cushman M, Das SR, de Ferranti S, Despres JP, Fullerton HJ, Howard VJ, Huffman MD, Isasi CR, Jimenez MC, Judd SE, Kissela BM, Lichtman JH, Lisabeth LD, Liu S, Mackey RH, Magid DJ, McGuire DK, Mohler ER 3rd, Moy CS, Muntner P, Mussolino ME, Nasir K, Neumar RW, Nichol G, Palaniappan L, Pandey DK, Reeves MJ, Rodriguez CJ, Rosamond W, Sorlie PD, Stein J, Towfighi A, Turan TN, Virani SS, Woo D, Yeh RW, Turner MB, Writing Group, M. (2016) American heart association statistics, and S. stroke statistics, heart disease and stroke statistics-2016 update: a report from the American heart association. Circulation 133(4):38-360

4. Braunwald E, Morrow DA (2013) Unstable angina: is it time for a requiem? Circulation 127(24):2452-2457

5. Reichlin T, Twerenbold R, Maushart C, Reiter M, Moehring B, Schaub N, Balmelli C, Rubini Gimenez M, Hoeller R, Sakarikos K, Drexler B, Haaf P, Osswald S, Mueller C (2013) Risk stratification in patients with unstable angina using absolute serial changes of 3 high-sensitive troponin assays. Am Heart J 165(3):371-8e3

6. Reichlin T, Twerenbold R, Reiter M, Steuer S, Bassetti S, Balmelli C, Winkler K, Kurz S, Stelzig C, Freese M, Drexler B, Haaf P, Zellweger C, Osswald S, Mueller C (2012) Introduction of highsensitivity troponin assays: impact on myocardial infarction incidence and prognosis. Am J Med 125(12):1205-1213

7. Thygesen K, Alpert JS, Jaffe AS, Chaitman BR, Bax JJ, Morrow DA, White HD, and I. Executive Group on behalf of the Joint European Society of Cardiology/American College of Cardiology/ 
American Heart Association/World Heart Federation Task Force for the Universal Definition of Myocardial (2018) Fourth universal definition of myocardial infarction (2018). Glob Heart 13(4):305-338

8. Roffi M, Patrono C, Collet JP, Mueller C, Valgimigli M, Andreotti F, Bax JJ, Borger MA, Brotons C, Chew DP, Gencer B, Hasenfuss G, Kjeldsen K, Lancellotti P, Landmesser U, Mehilli J, Mukherjee D, Storey RF, Windecker S, E.S.C.S.D. Group (2016) 2015 ESC guidelines for the management of acute coronary syndromes in patients presenting without persistent ST-segment elevation: task force for the management of acute coronary syndromes in patients presenting without persistent ST-segment elevation of the European Society of Cardiology (ESC). Eur Heart J 37(3):267-315

9. Chapman AR, Adamson PD, Shah ASV, Anand A, Strachan FE, Ferry AV, Lee KK, Berry C, Findlay I, Cruikshank A, Reid A, Gray A, Collinson PO, Apple F, McAllister DA, Maguire D, Fox KAA, Vallejos CA, Keerie C, Weir CJ, Newby DE, Mills NL, High SI (2020) High-sensitivity cardiac troponin and the universal definition of myocardial infarction. Circulation 141(3):161-171

10. Kadesjo E, Roos A, Siddiqui AJ, Sartipy U, Holzmann MJ (2021) Treatment with cardiovascular medications: prognosis in patients with myocardial injury. J Am Heart Assoc 10(1):e017239

11. McCarthy CP, Olshan DS, Rehman S, Jones-O'Connor M, Murphy S, Cohen JA, Singh A, Vaduganathan M, Januzzi JL Jr, Wasfy JH (2021) Cardiologist evaluation of patients with type 2 myocardial infarction. Circ Cardiovasc Qual Outcomes 14(1):e007440

12. Collet JP, Thiele H, Barbato E, Barthelemy O, Bauersachs J, Bhatt DL, Dendale P, Dorobantu M, Edvardsen T, Folliguet T, Gale CP, Gilard M, Jobs A, Juni P, Lambrinou E, Lewis BS, Mehilli J, Meliga E, Merkely B, Mueller C, Roffi M, Rutten FH, Sibbing D, Siontis GCM, E.S.C.S.D. Group (2021) 2020 ESC guidelines for the management of acute coronary syndromes in patients presenting without persistent ST-segment elevation. Eur Heart J 42(14):1289-1367

13. Ibanez, B., S. James, S. Agewall, M.J. Antunes, C. BucciarelliDucci, H. Bueno, A.L.P. Caforio, F. Crea, J.A. Goudevenos, S. Halvorsen, G. Hindricks, A. Kastrati, M.J. Lenzen, E. Prescott, M. Roffi, M. Valgimigli, C. Varenhorst, P. Vranckx, P. Widimsky, 2017 ESC Guidelines for the management of acute myocardial infarction in patients presenting with ST-segment elevation: The Task Force for the management of acute myocardial infarction in patients presenting with ST-segment elevation of the European Society of Cardiology (ESC). Eur Heart J, 2018. 39(2):119-177.

14. Saaby L, Poulsen TS, Hosbond S, Larsen TB, Pyndt Diederichsen AC, Hallas J, Thygesen K, Mickley H (2013) Classification of myocardial infarction: frequency and features of type 2 myocardial infarction. Am J Med 126(9):789-797

15. Lombardi CM, Carubelli V, Iorio A, Inciardi RM, Bellasi A, Canale C, Camporotondo R, Catagnano F, Dalla Vecchia LA, Giovinazzo S, Maccagni G, Mapelli M, Margonato D, Monzo L, Nuzzi V, Oriecuia C, Peveri G, Pozzi A, Provenzale G, Sarullo F, Tomasoni D, Ameri P, Gnecchi M, Leonardi S, Merlo M, Agostoni P, Carugo S, Danzi GB, Guazzi M, La Rovere MT, Mortara A, Piepoli M, Porto I, Sinagra G, Volterrani M, Specchia C, Metra M, Senni M (2020) Association of troponin levels with mortality in Italian patients hospitalized with coronavirus disease 2019: results of a multicenter study. JAMA Cardiol 5(11):1274-1280

16. Raphael CE, Roger VL, Sandoval Y, Singh M, Bell M, Lerman A, Rihal CS, Gersh BJ, Lewis B, Lennon RJ, Jaffe AS, Gulati $\mathrm{R}$ (2020) Incidence, trends, and outcomes of type 2 myocardial infarction in a community cohort. Circulation 141(6):454-463

17. Chapman AR, Shah ASV, Lee KK, Anand A, Francis O, Adamson P, McAllister DA, Strachan FE, Newby DE, Mills NL (2018) Long-term outcomes in patients with type 2 myocardial infarction and myocardial injury. Circulation 137(12):1236-1245
18. Smilowitz NR, Subramanyam P, Gianos E, Reynolds HR, Shah B, Sedlis SP (2018) Treatment and outcomes of type 2 myocardial infarction and myocardial injury compared with type 1 myocardial infarction. Coron Artery Dis 29(1):46-52

19. Stein GY, Herscovici G, Korenfeld R, Matetzky S, Gottlieb S, Alon D, Gevrielov-Yusim N, Iakobishvili Z, Fuchs S (2014) TypeII myocardial infarction-patient characteristics, management and outcomes. PLoS One 9(1):e84285

20. Gaggin HK, Liu Y, Lyass A, van Kimmenade RR, Motiwala SR, Kelly NP, Mallick A, Gandhi PU, Ibrahim NE, Simon ML, Bhardwaj A, Belcher AM, Harisiades JE, Massaro JM, D'Agostino RB Sr, Januzzi JL Jr (2017) Incident type 2 myocardial infarction in a cohort of patients undergoing coronary or peripheral arterial angiography. Circulation 135(2):116-127

21. de Lemos JA, Newby LK, Mills NL (2019) A proposal for modest revision of the definition of type 1 and type 2 myocardial infarction. Circulation 140(22):1773-1775

22. McCarthy CP, Kolte D, Kennedy KF, Vaduganathan M, Wasfy JH, Januzzi JL Jr (2021) Patient characteristics and clinical outcomes of type 1 versus type 2 myocardial infarction. J Am Coll Cardiol 77(7):848-857

23. Gupta S, Vaidya SR, Arora S, Bahekar A, Devarapally SR (2017) Type 2 versus type 1 myocardial infarction: a comparison of clinical characteristics and outcomes with a meta-analysis of observational studies. Cardiovasc Diagn Ther 7(4):348-358

24. Arora S, Strassle PD, Qamar A, Wheeler EN, Levine AL, Misenheimer JA, Cavender MA, Stouffer GA, Kaul P (2018) Impact of type 2 myocardial infarction (MI) on hospital-level MI outcomes: implications for quality and public reporting. J Am Heart Assoc 7(7):e008661

25. DeFilippis AP, Chapman AR, Mills NL, de Lemos JA, ArbabZadeh A, Newby LK, Morrow DA (2019) Assessment and treatment of patients with type 2 myocardial infarction and acute nonischemic myocardial injury. Circulation 140(20):1661-1678

26. Horiuchi Y, Wettersten N, Patel MP, Mueller C, Neath SX, Christenson RH, Morgenthaler NG, McCord J, Nowak RM, Vilke GM, Daniels LB, Hollander JE, Apple FS, Cannon CM, Nagurney JT, Schreiber D, deFilippi C, Hogan C, Diercks DB, Headden G, Limkakeng AT Jr, Anand I, Wu AHB, Ebmeyer S, Jaffe AS, Peacock WF, Maisel A (2020) Biomarkers enhance discrimination and prognosis of type 2 myocardial infarction. Circulation 142(16): 1532-1544

27. Neumann JT, Sorensen NA, Rubsamen N, Ojeda F, Renne T, Qaderi V, Teltrop E, Kramer S, Quantius L, Zeller T, Karakas M, Blankenberg S, Westermann D (2017) Discrimination of patients with type 2 myocardial infarction. Eur Heart J 38(47):3514-3520

28. Kaul P, Fu Y, Chang WC, Harrington RA, Wagner GS, Goodman SG, Granger CB, Moliterno DJ, Van de Werf F, Califf RM, Topol EJ, Armstrong PW, Paragon A, G.I.I.P.I.I.A.f.t.R.o.A.G.O. Network (2001) Prognostic value of ST segment depression in acute coronary syndromes: insights from PARAGON-A applied to GUSTO-IIb. PARAGON-A and GUSTO IIb. Investigators platelet $\mathrm{IIb} / \mathrm{III}$ antagonism for the reduction of acute global organization network. J Am Coll Cardiol 38(1):64-71

29. Sandoval Y, Jaffe AS (2019) Type 2 myocardial infarction: JACC review topic of the week. J Am Coll Cardiol 73(14):1846-1860

30. Sandoval Y, Thygesen K (2017) Myocardial infarction type 2 and myocardial injury. Clin Chem 63(1):101-107

31. Sandoval Y, Thordsen SE, Smith SW, Schulz KM, Murakami MM, Pearce LA, Apple FS (2014) Cardiac troponin changes to distinguish type 1 and type 2 myocardial infarction and 180-day mortality risk. Eur Heart J Acute Cardiovasc Care 3(4):317-325

32. Gard A, Lindahl B, Batra G, Hadziosmanovic N, Hjort M, Szummer KE, Baron T (2018) Interphysician agreement on subclassification of myocardial infarction. Heart 104(15):1284-1291 
33. Lambrecht S, Sarkisian L, Saaby L, Poulsen TS, Gerke O, Hosbond S, Diederichsen ACP, Thygesen K, Mickley H (2018) Different causes of death in patients with myocardial infarction type 1, type 2, and myocardial injury. Am J Med 131(5):548-554

34. Saaby L, Poulsen TS, Diederichsen AC, Hosbond S, Larsen TB, Schmidt H, Gerke O, Hallas J, Thygesen K, Mickley H (2014) Mortality rate in type 2 myocardial infarction: observations from an unselected hospital cohort. Am J Med 127(4):295-302

35. Vergallo R, Porto I, D’Amario D, Annibali G, Galli M, Benenati S, Bendandi F, Migliaro S, Fracassi F, Aurigemma C, Leone AM, Buffon A, Burzotta F, Trani C, Niccoli G, Liuzzo G, Prati F, Fuster V, Jang IK, Crea F (2019) Coronary atherosclerotic phenotype and plaque healing in patients with recurrent acute coronary syndromes compared with patients with long-term clinical stability: an in vivo optical coherence tomography study. JAMA Cardiol 4(4):321-329

36. Lindahl B, Baron T, Erlinge D, Hadziosmanovic N, Nordenskjold A, Gard A, Jernberg T (2017) Medical therapy for secondary prevention and long-term outcome in patients with myocardial infarction with nonobstructive coronary artery disease. Circulation 135(16): 1481-1489

37. Mehta SR, Wood DA, Storey RF, Mehran R, Bainey KR, Nguyen H, Meeks B, Di Pasquale G, Lopez-Sendon J, Faxon DP, Mauri L, Rao SV, Feldman L, Steg PG, Avezum A, Sheth T, PinillaEcheverri N, Moreno R, Campo G, Wrigley B, Kedev S, Sutton A, Oliver R, Rodes-Cabau J, Stankovic G, Welsh R, Lavi S, Cantor WJ, Wang J, Nakamya J, Bangdiwala SI, Cairns JA, C.T.S. Committee, and Investigators (2019) Complete revascularization with multivessel PCI for myocardial infarction. N Engl J Med 381(15):1411-1421

38. White HD (2021) adding insult to injury: are there treatments for myocardial injury and type 2 myocardial infarction? J Am Heart Assoc 10(1):e019796

39. Lambrakis K, French JK, Scott IA, Briffa T, Brieger D, Farkouh ME, White H, Chuang AM, Tiver K, Quinn S, Kaambwa B, Horsfall M, Morton E, Chew DP (2019) The appropriateness of coronary investigation in myocardial injury and type 2 myocardial infarction (ACT-2): a randomized trial design. Am Heart J 208:11-20

40. Chapman AR, Sandoval Y (2021) Type 2 myocardial infarction: evolving approaches to diagnosis and risk-stratification. Clin Chem 67(1):61-69

41. Mohamed MO, Contractor T, Abramov D, Parwani P, Michos ED, Fischman D, Alraies MC, Bagur R, Mamas MA (2021) Sex-based differences in prevalence and outcomes of common acute conditions associated with type 2 myocardial infarction. Am J Cardiol $147: 8$

42. Hung J, Roos A, Kadesjo E, McAllister DA, Kimenai DM, Shah ASV, Anand A, Strachan FE, Fox KAA, Mills NL, Chapman AR, Holzmann MJ (2020) Performance of the GRACE 2.0 score in patients with type 1 and type 2 myocardial infarction. Eur Heart J 42:2552

43. Murphy SP, McCarthy CP, Cohen JA, Rehman S, Jones-O'Connor M, Olshan DS, Singh A, Vaduganathan M, Cui J, Januzzi JL Jr, Wasfy JH (2020) Application of the GRACE, TIMI, and TAR RACO risk scores in type 2 myocardial infarction. J Am Coll Cardiol 75(3):344-345

44. Okamatsu K, Takano M, Sakai S, Ishibashi F, Uemura R, Takano T, Mizuno K (2004) Elevated troponin T levels and lesion characteristics in non-ST-elevation acute coronary syndromes. Circulation 109(4):465-470

45. Nestelberger T, Boeddinghaus J, Gimenez MR, Lopez-Ayala P, Ratmann PD, Badertscher P, Wildi K, Wussler D, Koechlin L, Arslani K, Zimmermann T, Freese M, Rinderknecht T, Miro O, Martin-Sanchez FJ, Kawecki D, Geigy N, Keller D, Twerenbold $\mathrm{R}$, Muller C, A. Investigators (2021) Direct comparison of highsensitivity cardiac troponin $\mathrm{T}$ and $\mathrm{I}$ in the early differentiation of type 1 vs. type 2 myocardial infarction. Eur Heart J Acute Cardiovasc Care

46. Summers SM, Long B, April MD, Koyfman A, Hunter CJ (2018) High sensitivity troponin: the Sisyphean pursuit of zero percent miss rate for acute coronary syndrome in the ED. Am J Emerg Med 36(6):1088-1097

47. Galassi AR, Crea F, Araujo LI, Lammertsma AA, Pupita G, Yamamoto Y, Rechavia E, Jones T, Kaski JC, Maseri A (1993) Comparison of regional myocardial blood flow in syndrome $\mathrm{X}$ and one-vessel coronary artery disease. Am J Cardiol 72(2):134-139

48. Panting JR, Gatehouse PD, Yang GZ, Grothues F, Firmin DN, Collins P, Pennell DJ (2002) Abnormal subendocardial perfusion in cardiac syndrome $\mathrm{X}$ detected by cardiovascular magnetic resonance imaging. N Engl J Med 346(25):1948-1953

49. Maron DJ, Hochman JS, Reynolds HR, Bangalore S, O'Brien SM, Boden WE, Chaitman BR, Senior R, Lopez-Sendon J, Alexander KP, Lopes RD, Shaw LJ, Berger JS, Newman JD, Sidhu MS, Goodman SG, Ruzyllo W, Gosselin G, Maggioni AP, White HD, Bhargava B, Min JK, Mancini GBJ, Berman DS, Picard MH, Kwong RY, Ali ZA, Mark DB, Spertus JA, Krishnan MN, Elghamaz A, Moorthy N, Hueb WA, Demkow M, Mavromatis K, Bockeria O, Peteiro J, Miller TD, Szwed H, Doerr R, Keltai M, Selvanayagam JB, Steg PG, Held C, Kohsaka S, Mavromichalis S, Kirby R, Jeffries NO, Harrell FE Jr, Rockhold FW, Broderick S, Ferguson TB Jr, Williams DO, Harrington RA, Stone GW, Rosenberg Y, I.R. Group (2020) Initial invasive or conservative strategy for stable coronary disease. N Engl J Med 382(15):1395-1407

50. Subherwal S, Bach RG, Chen AY, Gage BF, Rao SV, Newby LK, Wang TY, Gibler WB, Ohman EM, Roe MT, Pollack CV Jr, Peterson ED, Alexander KP (2009) Baseline risk of major bleeding in non-ST-segment-elevation myocardial infarction: the CRUSADE (can rapid risk stratification of unstable angina patients suppress ADverse outcomes with early implementation of the ACC/AHA guidelines) bleeding score. Circulation 119(14):1873-1882

51. Urban P, Mehran R, Colleran R, Angiolillo DJ, Byrne RA, Capodanno D, Cuisset T, Cutlip D, Eerdmans P, Eikelboom J, Farb A, Gibson CM, Gregson J, Haude M, James SK, Kim HS, Kimura T, Konishi A, Laschinger J, Leon MB, Magee PFA, Mitsutake Y, Mylotte D, Pocock S, Price MJ, Rao SV, Spitzer E, Stockbridge N, Valgimigli M, Varenne O, Windhoevel U, Yeh RW, Krucoff MW, Morice MC (2019) Defining high bleeding risk in patients undergoing percutaneous coronary intervention: a consensus document from the academic research consortium for high bleeding risk. Eur Heart J 40(31):2632-2653

Publisher's Note Springer Nature remains neutral with regard to jurisdictional claims in published maps and institutional affiliations. 